\title{
Significados atribuídos por psicólogos hospitalares às relações entre profissionais de saúde na comunicação de más notícias
}

\author{
Meanings attributed by hospital psychologists to the relations between health professionals in the \\ communication of bad news
}

Significados atribuidos por psicólogos hospitalarios a las relaciones entre profesionales de la salud en la comunicación de malas noticias

\section{Resumo}

O presente estudo teve como objetivo compreender significados atribuídos por psicólogos hospitalares às relações entre profissionais de saúde de diferentes áreas em situações de comunicação de más notícias. Trata-se de um estudo qualitativo, cujos participantes foram 17 psicólogos hospitalares que atuavam em hospitais-gerais públicos brasileiros. O número de participantes foi determinado com base no critério de saturação. A coleta de dados foi desenvolvida a partir da realização de uma entrevista semidirigida e audiogravada com cada participante. As transcrições das audiogravações foram submetidas à análise de conteúdo. Verificou-se que os significados que os participantes atribuíram às relações que profissionais de saúde de diferentes áreas estabelecem entre si em situações de comunicação de más notícias se ancoraram em um parâmetro temporal, em torno do qual foram demarcadas responsabilidades específicas que estimulam distanciamentos e dificultam aproximações, sobretudo entre médicos e psicólogos hospitalares. Ademais, alguns participantes valorizaram o trabalho colaborativo, mas não sua materialização por meio da integração prática de saberes especializados. O presente estudo permite a circunscrição de entraves à interdependência que somente poderão ser superados se todos os profissionais de saúde encararem o sofrimento psíquico desencadeado nas situações de comunicação de más notícias como uma urgência subjetiva.

Palavras-chave: Profissionais da saúde; Comunicação em saúde; Relações interprofisssionais; Pesquisa qualitativa.

\begin{abstract}
This study aimed to understand meanings attributed by hospital psychologists to the relations between health professionals from different areas in situations of bad news communication. This is a qualitative study, whose participants were 17 hospital psychologists who worked in Brazilian public general hospitals. The number of participants was determined through saturation criterion. Data collection was carried out by conducting a semidirected and audio-recorded interview with each participant. Transcripts of recordings were submitted to content analysis. It was found that the meanings attributed to the participants to the relations that health professionals from different areas establish among themselves in situations of bad news communication were anchored in a temporal parameter, around which were demarcated specific responsibilities that encourage distancing and make approximations difficult, especially between physicians and hospital psychologists. Furthermore, some participants valued collaborative work, but not its materialization through the practical integration of specialized knowledge. This study allows the circumscription of obstacles to interdependence that can only be overcome if all health professionals face the psychological suffering triggered in situations of bad news communication as a subjective urgency.
\end{abstract}

Keywords: Health professionals; Health communication; Interprofessional relations; Qualitative research.

\section{Resumen}

Este estudio tuvo como objetivo comprender los significados atribuidos por psicólogos hospitalarios a las relaciones entre profesionales de la salud de diferentes áreas en situaciones de comunicación de malas noticias. Se trata de un 
estudio cualitativo, cuyos participantes fueron 17 psicólogos hospitalarios que trabajaban en hospitales generales públicos brasileños. El número de participantes se determinó con base en el criterio de saturación. La recolección de datos se llevó a cabo mediante la realización de una entrevista semidirigida y grabada en audio con cada participante. Las transcripciones de las grabaciones se sometieron a análisis de contenido. Se encontró que los significados que los participantes atribuían a las relaciones que los profesionales de la salud de diferentes áreas establecen entre ellos en situaciones de comunicación de malas noticias estaban anclados en un parámetro temporal, en torno al cual se delimitaron responsabilidades específicas que fomentan distanciamientos y dificultan aproximaciones, principalmente entre médicos y psicólogos hospitalarios. Además, algunos participantes valoraron el trabajo colaborativo, pero no su materialización a través de la integración práctica de conocimientos especializados. El presente estudio permite circunscribir obstáculos a la interdependencia que solo pueden superarse si todos los profesionales de la salud miraren el sufrimiento psicológico desencadenado en situaciones de comunicación de malas noticias como una urgencia subjetiva.

Palabras clave: Profesionales de la salud; Comunicación en salud; Relaciones interprofesionales; Investigación cualitativa.

\section{Introdução}

$\mathrm{Na}$ atualidade, encontra-se amplamente difundido o entendimento de que o trabalho em equipe é fundamental para fazer frente ao desafio da integralidade da atenção em saúde (Baquião et al., 2021; Rosen et al., 2018). Porém, como alertam Peduzzi et al. (2020), frequentemente as relações entre profissionais de saúde de diferentes áreas - em hospitais, sobretudo são marcadas por uma abertura bastante restrita à interação e à colaboração, o que dificulta o estabelecimento de um objetivo assistencial comum e conduz a ações fragmentadas e pouco efetivas. Tal fato indica que muitos deles preferem desempenhar suas atividades com elevado grau de autonomia e reduzido nível de interdependência, ainda acompanhando as referidas autoras.

A comunicação de más notícias - definida como processo por meio do qual profissionais de saúde reportam a pacientes e/ou a seus familiares eventos que ameaçam a continuidade da vida - se afigura como uma das situações em que o trabalho em equipe é particularmente importante (Amorim et al., 2021; Souto \& Schulze, 2019; Aein \& Delaram, 2014). Ocorre que, por sua natureza relacional, a comunicação de más notícias não pode ser reduzida a um procedimento fundamentado em um único saber especializado, conforme adverte Krieger (2017). Na rotina de hospitais brasileiros, todavia, comumente médicos são os únicos responsáveis por tal atividade, embora não se sintam capacitados para tanto por compreenderem que não conseguem responder adequadamente às questões emocionais suscitadas pela ação comunicacional (Biazar et al, 2019; Vogel et al., 2019; Monteiro \& Quintana, 2016).

Nesse cenário, as contribuições que outros profissionais de saúde - psicólogos hospitalares, inclusive - teriam a oferecer acabam sendo subutilizadas, o que tende a impactar negativamente na qualidade da assistência em saúde (Souto \& Schulze, 2019). Parece razoável afirmar que tal problema deriva, ao menos em parte, dos significados atribuídos às relações que se estabelecem entre profissionais de saúde de diferentes áreas em situações de comunicação de más notícias. Afinal, Turato (2005) assevera que é em torno dos significados sobre experiências individuais ou coletivas que as pessoas organizam as mais variadas esferas de suas vidas. E o referido autor acrescenta que as pesquisas qualitativas se distinguem por visarem, justamente, a compreensão de significados.

Contudo, a revisão da literatura brasileira empreendida por Gallego, Gomes e Peres (2021) constatou que, no país, são escassas as pesquisas qualitativas consagradas especificamente aos significados que profissionais de saúde de diferentes áreas atribuem às relações que estabelecem entre si em situações de comunicação de más notícias. Além disso, as referidas autoras verificaram que a participação de psicólogos hospitalares tem sido preterida na exploração desse tema, e assim delimita-se outra importante lacuna, considerando que tanto quem comunica quanto quem recebe uma má notícia relativa à saúde tende a vivenciar algum nível de sofrimento emocional, o que evidencia a importância de intervenções psicológicas antes, durante e/ou após a ação comunicacional (Aguado \& Cuesta, 2019). Diante do exposto, o presente estudo teve como objetivo compreender 
significados atribuídos por psicólogos hospitalares às relações entre profissionais de saúde de diferentes áreas em situações de comunicação de más notícias.

\section{Metodologia}

Trata-se de um estudo qualitativo, o qual se afigura como um recorte de uma pesquisa mais ampla e contou com a participação de 17 psicólogos hospitalares que, à época da coleta de dados, atuavam em hospitais-gerais públicos brasileiros e possuíam experiência quanto à comunicação de más notícias. A maioria dos participantes era do sexo feminino (n=16) e o tempo médio de experiência no cargo foi de 12 anos. Deve-se destacar também que o número de participantes foi determinado com base no critério de saturação, observando-se as recomendações de Fontanella, Ricas e Turato (2008) para pesquisas qualitativas no campo da saúde.

A coleta de dados foi desenvolvida a partir da realização de uma entrevista semidirigida com cada participante. Tal instrumento tem a vantagem de proporcionar ao pesquisador, por meio da definição de um pequeno número de perguntas, um guia temático que introduz os tópicos da conversação e, ao mesmo tempo, permite que os participantes se expressem com grande liberdade a respeito (Fontanella et al., 2006). As entrevistas ocorreram presencialmente, em espaços reservados no próprio local de trabalho dos participantes, e duraram, em média, 20 minutos. Ressalte-se ainda que a coleta de dados foi gravada em áudio e que todos os cuidados éticos necessários foram observados, sendo que a pesquisa que originou o presente estudo foi devidamente aprovada por um Comitê de Ética em Pesquisa.

As gravações em áudio foram transcritas de modo literal e integral. Na sequência, procedeu-se a análise de conteúdo, de acordo com as diretrizes preconizadas por Campos e Turato (2009) quanto ao emprego de tal técnica. Dessa forma, dois pesquisadores, após a leitura flutuante das transcrições, configuraram categorias preliminares levando em conta critérios de relevância mediante a identificação de conteúdos latentes e manifestos. Em um segundo momento, as categorias preliminares foram discutidas em uma reunião do respectivo grupo de pesquisa, considerando que esse procedimento conduz à maximização da validade de dados em pesquisas qualitativas (Faria-Schutzer et al., 2015). Norteados pelos subsídios proporcionados por essa reunião, os dois pesquisadores revisaram as categoriais preliminares e, assim, chegaram às versões definitivas.

\section{Resultados}

Na pesquisa da qual deriva o presente estudo foram configuradas, no total, três categorias. A seguir, serão reportados os resultados concernentes a uma delas, selecionada por responder ao objetivo aqui visado. Tal categoria foi intitulada "Segundos socorros" - em alusão ao termo "primeiros socorros", que designa, genericamente, uma ampla gama de cuidados imediatos prestados para evitar o agravamento da condição de saúde de uma pessoa - e reúne relatos emblemáticos dos significados que os participantes atribuíram às relações que profissionais de saúde de diferentes áreas estabelecem entre si em situações de comunicação de más notícias. É preciso esclarecer que os referidos significados se ancoraram em um parâmetro temporal, em torno do qual foram demarcadas responsabilidades específicas que estimulam distanciamentos e dificultam aproximações. Os Relatos 1 e 2 ilustram certos aspectos desse achado por realçarem que, para a maioria dos participantes, os psicólogos hospitalares deveriam intervir, de preferência, apenas após a ação comunicacional conduzida por outros profissionais de saúde.

Relato 1: "Eu acredito que [o psicólogo hospitalar deve] oferecer condições para que a pessoa possa [...] expressar aquela dor e aquele sofrimento [após receber uma má notícia] sem ficar exposta [...]. [O psicólogo hospitalar deve ser] uma presença [...], alguém em quem a pessoa pode se apoiar" (Participante 9).

Relato 2: "O tipo de intervenção que a gente [como psicólogo hospitalar] vai fazer [...] depende daquela escuta, né, 
que a pessoa vai demandar [após receber uma má notícia]. Às vezes, [ela] vai pedir pra dar uma mão, pra conversar, pra chorar, só pra ouvir, né, pra chamar alguém da família, pra estar lá junto no leito" (Participante 16).

Portanto, em consonância com os significados veiculados majoritariamente pelos participantes, os psicólogos hospitalares teriam a incumbência de "socorrer" alguém que, devido à natureza dramática das circunstâncias, necessitaria de sustentação a partir da escuta de questões subjetivas que emergem como consequência das informações que lhes são transmitidas por profissionais de saúde de outras áreas. Já os médicos seriam os principais encarregados da primeira etapa do processo de comunicação de más notícias, cujo intento consistiria em apresentar ao paciente - e/ou à sua família - dados preocupantes sobre seu estado de saúde, também de acordo com a maioria dos participantes. O Relato 3 constitui um exemplo quanto a esse posicionamento, embora dele se depreenda um tom de indignação: "Sempre é assim mesmo, eles [os médicos] falam a notícia e se retiram da sala, do ambiente em que está [sic], e falam: 'agora você pode conversar com o psicólogo"” (Participante 17).

Em suma: para os participantes, os psicólogos hospitalares, de modo geral, contribuiriam com a segunda etapa da comunicação de más notícias, no sentido de manejar o impacto psicológico da primeira etapa. Alguns participantes acentuaram as linhas divisórias do processo em pauta, dando a entender que preferem resguardá-las, o que, a rigor, parece culminar na descaracterização do trabalho em equipe, conforme o Relato 4 sugere: "Eu não acompanho a transmissão da má notícia. Eu vou trabalhar depois a demanda que o paciente vai me trazer" (Participante 2). Outros participantes sinalizaram compreender que uma maior interação entre os profissionais de saúde como um todo a propósito da comunicação de más notícias - com foco na negociação de um objetivo assistencial comum - seria benéfica. O Relato 5 exemplifica tal achado, e também aponta que os psicólogos hospitalares poderiam liderar um movimento nessa direção: "[O psicólogo hospitalar precisa] começar a fazer acordos com a equipe, né, pensar em possibilidades de fazer com que a situação não seja tão trágica" (Participante 5).

Ainda nessa perspectiva, foi citada por alguns participantes a relevância do diálogo com os profissionais de saúde de outras áreas como forma de preparação para a comunicação de más notícias - particularmente a fim de estimular a disponibilidade afetiva diante das necessidades apresentadas por pacientes e/ou familiares - e para viabilizar, na sequência, o manejo situacional apropriado, acompanhando os Relatos 6 e 7, respectivamente. O Relato 8, por seu turno, acentua a necessidade de corresponsabilização no tocante à abordagem de demandas de ordem emocional, observando-se, porém, os limites das competências de cada profissão. Logo, nesse conjunto de relatos se nota certa valorização do trabalho colaborativo, mas não sua materialização por meio da integração prática de saberes especializados.

Relato 6: "Acho que a gente [profissionais de saúde como um todo] tem que ter uma preparação para poder ter tranquilidade para acolher o sofrimento do outro. Como que a gente se prepara para algo dessa natureza? [...] É estar sempre discutindo alguns casos dificeis, com a equipe de modo geral" (Participante 10).

Relato 7: "Ele [paciente vítima de queimaduras] dizia que se mataria. No setor de [pacientes] queimados não fica acompanhante para adultos. Mas eu articulei com a equipe para ficar um acompanhante. Então a namorada [do paciente] ficou, porque eu fiquei preocupada com essa ideação [suicida] dele, o que ele poderia fazer à noite sozinho, né?" (Participante 6).

Relato 8: "Não é um único indivíduo que vai resolver todas as questões. Não é só o psicólogo [hospitalar] que vai conseguir acolher as questões psíquicas. Se não houver uma equipe por trás para poder dar apoio também, cada um dentro do seu papel, da sua função" (Participante 12).

Em paralelo, foi apontado que uma circunscrição a um núcleo de saberes - psicológicos, no caso - poderia, conforme 
o Relato 9, se revelar proveitosa: "Eu acho que o psicólogo [hospitalar] [...] vive em uma solidão muito grande nesses casos [de comunicação de más notícias] [...] Deveria ter mais discussão entre os profissionais da Psicologia sobre o modo como fazemos a intervenção" (Participante 11). E é interessante mencionar que, realçando o desmembramento do processo de comunicação de más notícias, um único participante afirmou, com o Relato 10, que vinha desenvolvendo um novo modelo de atuação, em razão do qual interviria junto aos familiares antes da ação comunicacional: "Geralmente agora eu primeiro chamo a família, converso com a família, e depois chamo a equipe médica [para comunicar uma má notícia]" (Participante 3).

\section{Discussão}

Aguado e Cuesta (2019) defendem que psicólogos hospitalares podem contribuir tanto auxiliando a planejar e conduzir o processo de comunicação de más notícias quanto se encarregando do manejo de seu impacto psicológico. Todavia, os significados depreendidos dos relatos aqui veiculados indicam que, para a maioria dos participantes, a presença na ação comunicacional parece representar uma prerrogativa médica, sendo que os psicólogos hospitalares teriam um papel mais relevante a desempenhar somente após a mesma. E esse papel consistiria, em síntese, em proporcionar escuta ao sofrimento psíquico mobilizado pelas informações transmitidas pelos médicos.

Tal achado, em certo aspecto, se torna compreensível tendo em vista que, nos hospitais, os médicos, essencialmente, se ocupam do padecimento do corpo biológico e procuram reestabelecer o funcionamento do organismo, de acordo com Giglio-Jacquemot (2005). A autora acrescenta que, quando isso ocorre junto a um paciente que apresenta risco de morte iminente, geralmente caracteriza-se uma emergência médica, ao passo que uma urgência médica costuma ser delimitada por um quadro clínico agudo, com possibilidade de complicações mais graves, o qual exige um atendimento rápido, porém não necessariamente imediato. E caberia aos médicos distinguir uma situação da outra com base apenas na identificação de sinais e sintomas supostamente objetivos, conforme proporcionada por uma avaliação de natureza técnica.

Os psicólogos hospitalares, por outro lado, tipicamente intervêm em momentos de ruptura que suscitam intensas angústias e causam grande desestabilização psíquica. Como observaram Cordeiro e Miranda (2020), quando esses momentos inviabilizam a utilização de palavras para nomear os sentimentos e, assim, cria-se um vazio correlativo de uma espécie de suspensão do tempo, têm-se aquilo que alguns autores, a partir de princípios psicanalíticos, chamam de "urgência subjetiva". Diferentemente de uma urgência médica, portanto, uma urgência subjetiva se define com base em critérios singulares. Além disso, a uma urgência subjetiva se deve responder prontamente, mas sem a preocupação com a resolução imediata de um problema crítico que norteia o atendimento de uma urgência médica (Azevedo, 2018; Calazans \& Bastos, 2008; Muñoz et al., 2019).

Diante do exposto, é possível inferir que, para os participantes, o sofrimento psíquico desencadeado em pacientes e/ou em seus familiares nas situações de comunicação de más notícias deve ser encarado pelos psicólogos hospitalares como uma urgência subjetiva, embora nenhum deles tenha empregado tal termo durante a coleta de dados. Cabe acrescentar que o referido posicionamento dos participantes enfatiza que aos psicólogos hospitalares compete intervir sem a pretensão de erradicar rapidamente um estado penoso, de ordem emocional, no caso, distinguindo-os dos profissionais de saúde de outras áreas, pois a abordagem de uma urgência subjetiva precisa ser conduzida de modo a fomentar a gradativa superação da paralisia em um tempo presente (Cordeiro \& Miranda, 2020). E esse tipo de paralisia pode advir na segunda etapa do processo de comunicação de más notícias conforme delimitada pelos participantes.

Não obstante, é pertinente reforçar que, de uma forma ou de outra, o diálogo com os demais profissionais de saúde em prol da comunicação de más notícias foi valorizado pela maioria dos participantes do presente estudo. E isso é condizente com a ampliação do grau de contato recomendado pela Política Nacional de Humanização (Ministério da Saúde, 2004) a fim de viabilizar a superação das fronteiras existentes entre núcleos de conhecimentos e intensificar a corresponsabilidade entre a 
equipe de saúde, inclusive quanto à abordagem de questões subjetivas. Assim sendo, realçar a importância do trabalho de psicólogos hospitalares para o acolhimento do sofrimento psíquico vivenciado por pacientes e/ou familiares que recebem más notícias - conforme foi feito em muitos relatos - não isenta automaticamente dessa tarefa os profissionais de saúde de outras áreas.

Contudo, os participantes do presente estudo, de modo geral, se mostraram favoráveis ao trabalho colaborativo, mas não defenderam sua materialização a partir da integração prática de saberes especializados nas situações de comunicação de más notícias. Por essa razão, aparentemente estariam mais alinhados à interdisciplinaridade do que à interprofissionalidade, pois, na composição de tais termos, os sufixos "disciplinar" e "profissional", embora muitas vezes empregados como sinônimos, se distinguem por aludirem, respectivamente, ao plano teórico e ao plano concreto da atenção em saúde (Baquião et al., 2021). Logo, a interprofissionalidade pressupõe o compartilhamento estratégico de práticas, e não apenas o intercâmbio de conhecimentos, salvo, claro, no que diz respeito ao desempenho de atividades que constituem atribuições exclusivas de determinadas áreas, conforme D'amour e Oandasan (2005).

De qualquer maneira, acolher o sofrimento psíquico mobilizado em pacientes e/ou familiares após a comunicação de más notícias foi enfatizado por alguns participantes como uma atividade que deve ser assumida pelos profissionais de saúde em sua generalidade, independentemente da área. Revela-se, portanto, a adesão desses participantes em particular às diretrizes estabelecidas pelo Ministério da Saúde (2010) a propósito do acolhimento a ser exercitado no cotidiano, como postura ética e ferramenta tecnológica, pelos profissionais de saúde como um todo visando à qualificação da escuta, à produção de vínculos e à resolução das demandas de saúde da população. Além disso, vale frisar que o acolhimento é considerado um recurso imprescindível para a consecução dos objetivos da Política Nacional de Humanização (Ministério da Saúde, 2004).

\section{Considerações Finais}

O presente estudo permite a circunscrição de limites e possibilidades quanto às relações entre profissionais de saúde de diferentes áreas em situações de comunicação de más notícias de acordo com os significados atribuídos pelos participantes. Ocorre que, para a maioria deles, tais situações se desmembram em dois momentos contrastantes, o que determina uma divisão do trabalho em equipe - sobretudo entre médicos e psicólogos hospitalares - que espelha a distinção entre urgências e emergências médicas e urgências subjetivas, conforme aqui exposta. Porém, alguns participantes reconheceram que os demais profissionais de saúde são capazes de contribuir com a ação comunicacional, e também, eventualmente, com o acolhimento de suas repercussões emocionais.

Ainda assim, o intercâmbio de saberes, em detrimento do compartilhamento estratégico de práticas, foi apontado pelos participantes como o caminho mais exequível para tanto, e isso tende a impor entraves à interdependência. Parece razoável propor que esses entraves somente poderão ser superados se todos os profissionais de saúde encararem o sofrimento psíquico desencadeado nas situações de comunicação de más notícias como uma urgência subjetiva. Por outro lado, o presente estudo possui limites, e talvez o principal seja relativo ao fato de não problematizar diretamente o modelo medicalocêntrico vigente em muitos hospitais. Tal modelo já foi tematizado por outros autores, mas não especificamente quanto a seus desdobramentos para a comunicação de más notícias. Sugere-se, então, que pesquisas futuras se ocupem dessa empreitada.

Recomenda-se, outrossim, que pesquisadores interessados em explorar as relações entre profissionais de saúde de diferentes áreas em situações de comunicação de más notícias recorram à triangulação de dados oriundos de fontes variadas. Pode ser considerada uma opção potencialmente proveitosa nesse sentido a combinação de entrevistas semidirigidas e observações-participantes. Afinal, entrevistas semidirigidas se afiguram como instrumentos de grande relevância em pesquisas qualitativas, como, inclusive, o presente estudo atesta. E observações-participantes favorecem o entendimento, por parte do pesquisador, de certos aspectos das relações entre pessoas e instituições que dificilmente são captados de outra maneira, 
sobretudo no campo da saúde, como defendem Fernandes e Moreira (2013).

\section{Referências}

Aein, F., \& Delaram, M. (2014). Giving bad news: A qualitative research exploration. Iranian Red Crescent Medical Journal, 16(6), e8197. https://doi.org/10.5812/ircmj.8197

Aguado, M. P. Q., \& Cuesta, M. B. (2019). Aportaciones de la Psicología a la comunicación en el ámbito sanitario. Ciencias Psicológicas, 13(2), 317-332. https://doi.org/10.22235/cp.v13i2.1889

Amorim, C. B., Barlem, E. L. D., Piexak, D. R., Avancini, R. C., Lima, F. G., \& Alves, B. R. (2021). Comunicação de notícias difíceis: Facilidades, dificuldades e estratégias utilizadas pelos estudantes de Enfermagem na formação. Texto \& Contexto - Enfermagem, 30 , e20200044. https://doi.org/10.1590/1980-265X-TCE-2020-0044

Azevedo, E. (2018). Da pressa à urgência do sujeito: Psicanálise e urgência subjetiva. Analytica: Revista de Psicanálise, 7(13), 208-217. http://www.seer.ufsj.edu.br/index.php/analytica/article/view/3343/2107

Baquião, A. P. S. S., Guedes, L. M., Peres, R. S., \& Grincenkov, F. R. S. (2021). Interdisciplinarity and interprofessionality in teamwork: Perceptions of multiprofessional residents in Hospital Care. Acta Scientiarum - Health Sciences, 43, e54332. https://doi.org/10.4025/actascihealthsci.v43i1.54332

Biazar, G., Delpasand, K., Farzi, F., Sedighinejad, A., Mirmansouri, A., \& Atrkarroushan, Z. (2019). Breaking bad news: A valid concern among clinicians. Iranian Journal of Psychiatry, 14(3), 198-202. https://www.ncbi.nlm.nih.gov/pmc/articles/PMC6778609/pdf/IJPS-14-198.pdf

Calazans, R., \& Bastos, A. (2008). Urgência subjetiva e clínica psicanalítica. Revista Latinoamericana de Psicopatologia Fundamental, 11(4), 640-652. https://doi.org/10.1590/S1415-47142008000400010

Campos, C. J. G., \& Turato, E. R. (2009). Análise de conteúdo em pesquisas que utilizam metodologia clínico-qualitativa: Aplicação e perspectivas. Revista Latino-Americana de Enfermagem, 17(2), 259-264. https://doi.org/10.1590/S0104-11692009000200019

Cordeiro, S. N., \& Miranda, F. S. (2020). A vida por um fio: A escuta clínica entre a urgência subjetiva e a urgência médica. Estudos Interdisciplinares em Psicologia, 11(3), 132-145. https://doi.org/10.5433/2236-6407.2020v11n3suplp132

D'amour, D., \& Oandasan, I. (2005). Interprofessionality as the field of interprofessional practice and interprofessional education: An emerging concept. Journal of Interprofessional Care, 19(supl. 1), 8-20. https://doi.org/10.1080/13561820500081604

Faria-Schutzer, D. B., Surita, F. G., Alves, V. L., Vieira, C. M., \& Turato, E. R. (2015). Emotional experiences of obese women with adequate gestational weight variation: A qualitative study. PLoS One, 10(11), e0141879. https://doi.org/10.1371/journal.pone.0141879

Fernandes, F. M. B., \& Moreira, M. R. (2013). Considerações metodológicas sobre as possibilidades de aplicação da técnica de observação participante na Saúde Coletiva. Physis: Revista de Saúde Coletiva, 23(2), 511-529. https://doi.org/10.1590/S0103-73312013000200010

Fontanella, B. J. B., Campos, C. J. G., \& Turato, E. R. (2006). Coleta de dados na pesquisa clínico-qualitativa: Uso de entrevistas não-dirigidas de questões abertas por profissionais da saúde. Revista Latino-Americana de Enfermagem, 14(5), 812-820. https://doi.org/10.1590/S0104-11692006000500025

Fontanella, B. J. B., Ricas J. \& Turato E. R. (2008). Amostragem por saturação em pesquisas qualitativas em saúde: Contribuições teóricas. Cadernos de Saúde Pública, 24(1), 17-27. https://doi.org/10.1590/S0102-311X2008000100003

Gallego, P. B., Gomes, L. R. S., \& Peres, R. S. (2021). Comunicação de más notícias no contexto da atenção em saúde: Uma revisão integrativa. In B. A. D. Pestana, I. G. Souza, \& I. L. Perissato (Orgs.), Revisões em saúde coletiva: Tendências, recorrências e lacunas (pp. 123-136). Colab.

Giglio-Jacquemot, A. (2005). Urgências e emergências em saúde: Perspectivas de profissionais e usuários. Editora Fiocruz.

Ministério da Saúde (2004). Política Nacional de Humanização: A humanização como eixo norteador das práticas de atenção e gestão em todas as instâncias do Sistema Único de Saúde. Brasília: Ministério da Saúde. https://bvsms.saude.gov.br/bvs/publicacoes/humanizasus_2004.pdf

Ministério da Saúde (2010). Acolhimento nas práticas de produção de saúde. (2a ed.), Ministério da Saúde. https://bvsms.saude.gov.br/bvs/publicacoes/acolhimento_praticas_producao_saude.pdf

Monteiro, D. T., \& Quintana, A. M. (2016). A comunicação de más notícias na UTI: Perspectiva dos médicos. Psicologia: Teoria e Pesquisa, 32 (4), e324221. https://doi.org/10.1590/0102.3772e324221

Muñoz, N. M., Vilanova, A., Tenembaum, D., \& Velasco, L. B. (2019). O manejo da urgência subjetiva na universidade: Construindo estratégias de cuidado à saúde mental dos estudantes. Interação em Psicologia, 23(2), 177-183. https://doi.org/10.5380/psi.v23i02.58547

Peduzzi, M., Agreli, H. L. F., Silva, J. A. M., \& Souza, H. S. (2020). Trabalho em equipe: Uma revisita ao conceito e a seus desdobramentos no trabalho interprofissional. Trabalho, Educação e Saúde, 18(suppl. 1), e0024678. https://doi.org/10.1590/1981-7746-sol00246

Krieger, M. V. (2017). Comunicação de más notícias em saúde: Contribuições à discussão bioética através de uma nova ética das virtudes (Dissertação de mestrado). Fundação Oswaldo Cruz, Rio de Janeiro.

Rosen, M. A., DiazGranados, D., Dietz, A. S., Benishek, L. E., Thompson, D., Pronovost, P. J., \& Weaver, S. J. (2018). Teamwork in healthcare: Key discoveries enabling safer, high-quality care. The American Psychologist, 73(4), 433-450. https://doi.org/10.1037/amp0000298

Silva, M. J. P. (2012). Comunicação de más notícias. $O$ Mundo da Saúde, 36(1), 49-53. https://bvsms.saude.gov.br/bvs/artigos/mundo_saude/comunicacao_mas_noticias.pdf 
Research, Society and Development, v. 11, n. 2, e42911225688, 2022

(CC BY 4.0) | ISSN 2525-3409 | DOI: http://dx.doi.org/10.33448/rsd-v11i2.25688

Souto, D. C., \& Schulze, M. D. (2019). Profissionais de saúde e comunicação de más notícias: Experiências de uma unidade neonatal. Psicologia e Saúde, 11(3), 173-184. https://doi.org/10.20435/pssa.v0i0.690

Turato, E. R. (2005). Métodos qualitativos e quantitativos na área da saúde: Definições, diferenças e seus objetos de pesquisa. Revista de Saúde Pública, 39(3), 507-514. https://doi.org/10.1590/S0034-89102005000300025

Vogel, K. P., Silva, J. H. G., Ferreira, L. C., \& Machado, L. C. (2019). Comunicação de más notícias: Ferramenta essencial na graduação médica. Revista Brasileira de Educação Médica, 43(suppl 1), 314-321. https://doi.org/10.1590/1981-5271v43suplemento1-20180264 